OPEN ACCESS

Edited by:

Cristina Vassalle,

Gabriele Monasterio Tuscany

Foundation (CNR), Italy

Reviewed by:

Hopewell Nkosipendule Ntsinjana,

University of the Witwatersrand,

South Africa

Madhusudan Ganigara,

Donald and Barbara Zucker School of

Medicine at Hofstra-Northwell,

United States

*Correspondence:

Mohsen Sedighi

sedighi.mo@iums.ac.ir

orcid.org/0000-0003-0608-7840

Specialty section:

This article was submitted to

Pediatric Cardiology,

a section of the journal

Frontiers in Cardiovascular Medicine

Received: 02 July 2020 Accepted: 14 October 2020 Published: 12 November 2020

Citation:

Bigdelian H, Sedighi M, Sabri MR, Dehghan B, Pourmoghaddas Z, Saleh R, Sherafat A, Massoumi G, Rezaei MK and Shahbazi M (2020) Right Atrial Thrombus in a COVID-19

Child Treated Through Cardiac

Surgery.

Front. Cardiovasc. Med. 7:579522.

doi: 10.3389/fcvm.2020.579522

\title{
Right Atrial Thrombus in a COVID-19 Child Treated Through Cardiac Surgery
}

\author{
Hamid Bigdelian ${ }^{1}$, Mohsen Sedighi 2*, Mohammad Reza Sabri ${ }^{1}$, Bahar Dehghan ${ }^{1}$, \\ Zahra Pourmoghaddas ${ }^{3}$, Rana Saleh ${ }^{3}$, Alireza Sherafat ${ }^{4}$, Gholamreza Massoumi ${ }^{1}$, \\ Mohammad Kazem Rezaei ${ }^{1}$ and Masoud Shahbazi ${ }^{1}$ \\ ${ }^{1}$ Cardiovascular Research Institute, Isfahan University of Medical Sciences, Isfahan, Iran, ${ }^{2}$ Neuroscience Research Center, \\ Iran University of Medical Sciences, Tehran, Iran, ${ }^{3}$ Pediatric Department, School of Medicine, Isfahan University of Medical \\ Sciences, Isfahan, Iran, ${ }^{4}$ School of Medicine, University of Central Lancashire, Preston, United Kingdom
}

We herein report a case of large intracardiac thrombus in a child with SARS-CoV-2 infection (COVID-19). The diagnosis of COVID-19 was confirmed through HRCT and RT-PCR. Transthoracic echocardiography revealed a large thrombus in the right atrium treated successfully via cardiac surgery. The underlying mechanisms of this thrombus in the COVID-19 infection may be attributed to the hypercoagulation and inflammatory condition incurred by the COVID-19 virus.

Keywords: COVID-19, cardiac surgery, pediatric, thrombus-echocardiography, fever

\section{INTRODUCTION}

In December 2019, an outbreak of new viral pneumonia caused by the novel coronavirus (SARS-CoV-2) occurred in Wuhan, China (1). This virus is responsible for COVID-19 (Corona Virus Disease 2019) and can lead to different symptoms ranging from a mild viral disease to acute respiratory distress syndrome (ARDS), multi-organ failure, and death $(2,3)$. Although cardiovascular complications of COVID-19 has been well-described in the current literature, intracardiac thrombus complication in children has been rarely reported. Intracardiac thrombus caused by SARS-CoV-2 infection is a serious and life-threatening complication in COVID-19 patients (4) and the size of thrombus at the time of diagnosis correlates strongly with increased risk of thromboembolism and sudden death (5). Here, we describe a case report of large thrombus formation in the right atrium (RA) of a child with COVID-19 infection required emergency cardiac surgery, as the only preferred treatment to remove such a large thrombus. 


\section{CASE REPORT}

On 7 April 2020, an 11-years-old boy was admitted to the pediatric hospital in Esfahan with high fever, dyspnea, and skin rashes. Past medical history of patient showed that he was a known case of seizure disorder and neurodevelopmental delay, and had been under treatment with phenobarbital. Blood gas analysis on the admission time showed a $\mathrm{pH}$ of 7.27, $\mathrm{pCO}_{2}$ of $49.5 \mathrm{mmHg}, \mathrm{pO}_{2}$ of $44.8 \mathrm{mmHg}$, and oxygen saturation $\left(\mathrm{O}_{2}\right.$ Sat $)$ of $73 \%$. Also, his laboratory test results were as follows: white blood cell count (WBC) of $11.4 \times$ $10^{*}$ / $/ \mathrm{L}$ (reference range: $4.5-11$ ), C-reactive protein (CRP) 38 $\mathrm{mg} / \mathrm{dL}$ (reference range: up to 6), erythrocyte sedimentation

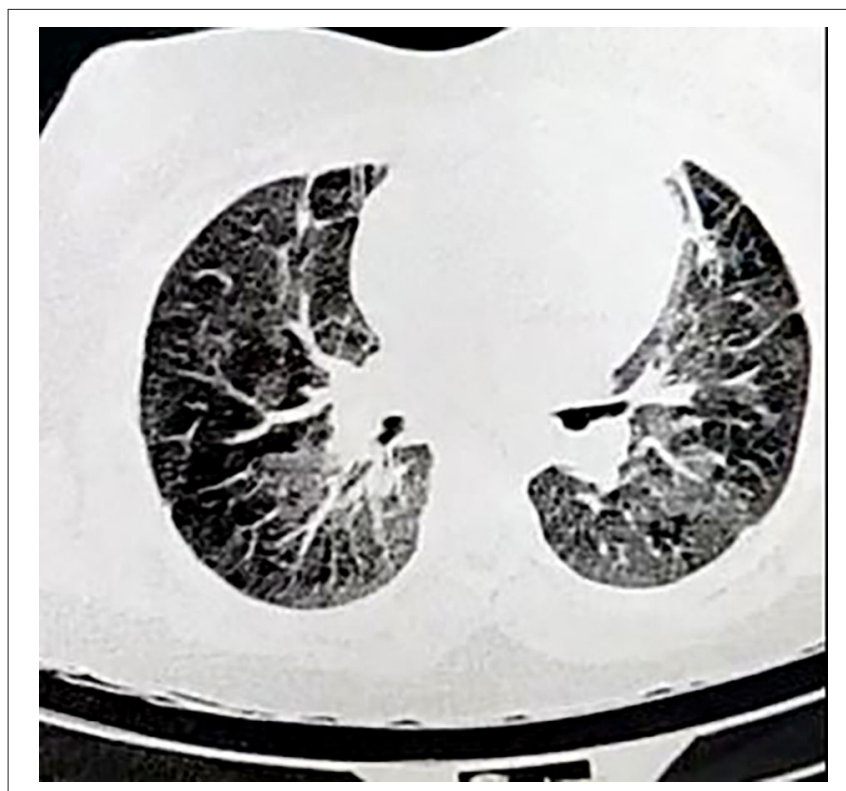

FIGURE 1 | High-resolution computed tomography (HRCT) of the lungs in COVID-19 child showing bilateral multiple patchy areas of ground glass opacities. rate (ESR) $38 \mathrm{~mm} / \mathrm{h}$ (reference range: up to 20), and lactate dehydrogenase (LDH) $427 \mathrm{U} / \mathrm{L}$ (reference range: 140-280). Chest x-ray and high-resolution computed tomography (HRCT) showed typical ground-glass opacitiesin both lungs, suggesting viral pneumonia (Figure 1). Throat swab samples analysis by reverse transcription-polymerase chain reaction (RT-PCR) confirmed COVID-19 infection and treatment of disease was started according to the Iranian pediatric protocol including hydroxychloroquine (5 $\mathrm{mg} / \mathrm{kg} /$ dose $)$, lopinavir /ritonavir (230 $\mathrm{mg} / \mathrm{m}^{2} /$ dose $)$, ceftriaxone $(75 \mathrm{mg} / \mathrm{kg} /$ dose $)$, and vancomycin $(10$ $\mathrm{mg} / \mathrm{kg} / \mathrm{dose}$ ) (6). On the $2 \mathrm{nd}$ day of hospitalization, he presented shortness of breathing and decreased level of consciousness (LOC) that led to intubation and he put on a ventilator. After extubation, Bi-level positive airway pressure (BiPAP) was administrated for respiratory support. Despite receiving drug treatment for COVID-19, his laboratory test results were significant for continuous leukocytosis and neutrophilia (Table 1). Because of persistent fever and tachycardia, pediatric cardiology consultation was requested and transthoracic echocardiography revealed a large mobile homogenous mass $(2.5$ $\times 1.5 \mathrm{~cm}$ ) on the tricuspid valve leaflet extended to the RA and right ventricle $(\mathrm{RV})$ with attachment to the tip of central venous catheter (CVC) that was in favor of thrombus or vegetation (Figure 2). Besides, trivial tricuspid regurgitation (TR) and trivial pulmonary insufficiency (PI) were found. Consequently, he was transferred to the Chamran Heart Center on 16 April 2020 for surgical intervention. A sternotomy with cardiopulmonary bypass was performed and intraoperative observations revealed a large thrombus in RA which was removed completely together with CVC while cardiac valves were preserved (Figure 3). After the surgery, he was admitted to the ICU, and the pediatric ward thereafter. Culture result of the thrombus showed no fungal and bacterial infection and also SARS-CoV-2 RNA was not detected in the mass. Histological analysis of the mass showed inflammatory infiltrate (mostly neutrophils) formation along with organizing thrombus and partial necrosis of tricuspid valve leaflet. He was discharged after 2 weeks in good health condition.

TABLE 1 | Laboratory test results of patient during the course of disease.

\begin{tabular}{|c|c|c|c|c|c|c|}
\hline Variables & Reference range & Admission & Day 5 & Day 10 & Before surgery & After surgery \\
\hline RBC count ( $\left.\times 10^{*} 12 / L\right)$ & $4.5-6.5$ & 3.84 & 4.04 & 4.13 & 3.88 & 4.63 \\
\hline Hematocrit (\%) & $41-51$ & 36.9 & 38.3 & 38.4 & 31.6 & 41.3 \\
\hline Hemoglobin (g/dL) & $13-17$ & 11.5 & 12.5 & 13.3 & 10.9 & 14.5 \\
\hline WBC (×10*9/L) & $4.5-11$ & 11.4 & 12.2 & 13.5 & 21.3 & 11.2 \\
\hline Lymphocyte count (\%) & $20-40$ & 27 & 15 & 16 & 15.1 & 17.5 \\
\hline Neutrophil count (\%) & $50-70$ & 66.4 & 78 & 72.1 & 75.7 & 72.9 \\
\hline Platelet count $\left(\times 10^{\star} 9 / \mathrm{L}\right)$ & $150-450$ & 252 & 279 & 247 & 400 & 238 \\
\hline PT (second) & $11-13$ & 13 & 12.8 & 12.2 & 17.1 & 16 \\
\hline PTT (second) & $26-45$ & 38 & 37 & 33 & 30 & 28 \\
\hline INR & $0.8-1.2$ & 1.1 & 1.1 & 1.1 & 1.4 & 1.2 \\
\hline Blood culture $(\times 3)$ & $+1-$ & - & - & - & - & - \\
\hline
\end{tabular}

RBC, red blood cell; WBC, white blood cell; PT, prothrombin time; PTT, partial thromboplastin time; INR, international normalized ratio. 


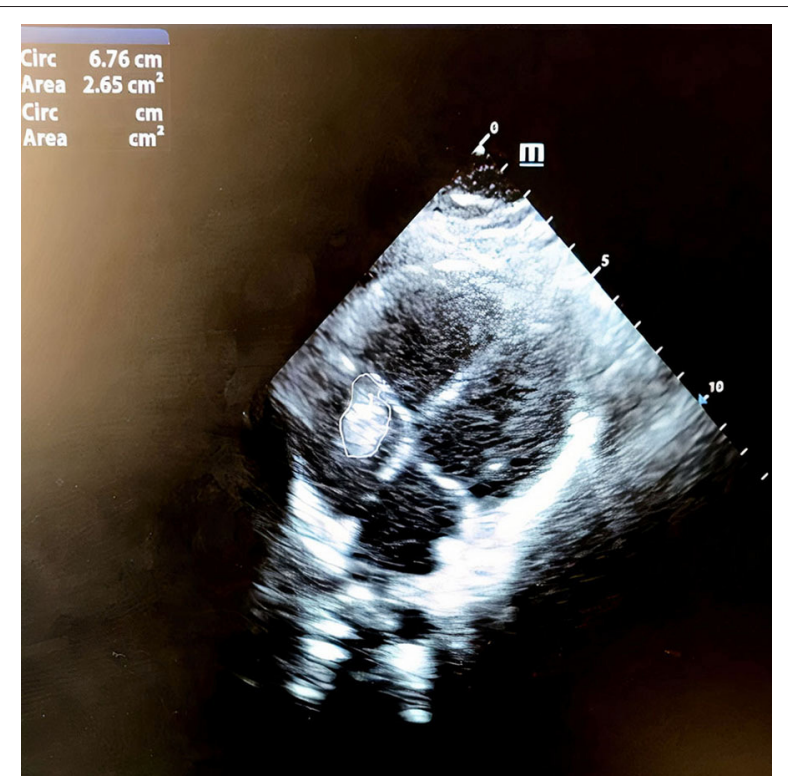

FIGURE 2 | Transthoracic echocardiogram showing a large $2.5 \times 1.5 \mathrm{~cm}$ mass on tricuspid valve leaflet protruded to the right atrium and right ventricle.

\section{DISCUSSION}

Accumulating evidence has indicated cardiovascular involvement of COVID-19 infection which can lead to the poor clinical prognosis of this disease. Discriminating between a cardiac or pulmonary cause of symptoms can be difficult because each may present mostly with dyspnea. Recent reports have shown that COVID-19 is associated with the increased incidence of cardiovascular complications including myocarditis, acute cardiac injury, and arrhythmias (7). Moreover, it has been demonstrated that inflammation and thrombosis mutually reinforce each other and COVID-19 infection can lead to the coagulopathy likely due to infection-induced inflammatory changes (8). Remarkable inflammation is observed in patients with COVID-19 infection presenting by elevated levels of IL-6, increased CRP and erythrocyte sedimentation rate (ESR). This inflammatory condition and subsequent activation of coagulation are the probable causes for the hypercoagulable state in COVID-19 infection (9). The intracardiac thrombus formation has rarely been described in the COVID-19 patients and our finding supports current reports that indicate higher thrombotic risk in COVID-19 patients $(10,11)$. The presence of a large thrombus in RA was an unexpected finding in our case because he had no history of heart disease or coagulopathy. Similar to the other reports, we believe that the bigger the size of the clot, the greater the chance that medical treatment will fail (12). Removal of such a large thrombus needs surgical intervention and surgical thrombectomy should be opted to treat large right atrial thrombus (more than $2 \mathrm{~cm}$ ) in the absence of any contraindication for surgery. In parallel with us, a literature review by Negulescu et al. has

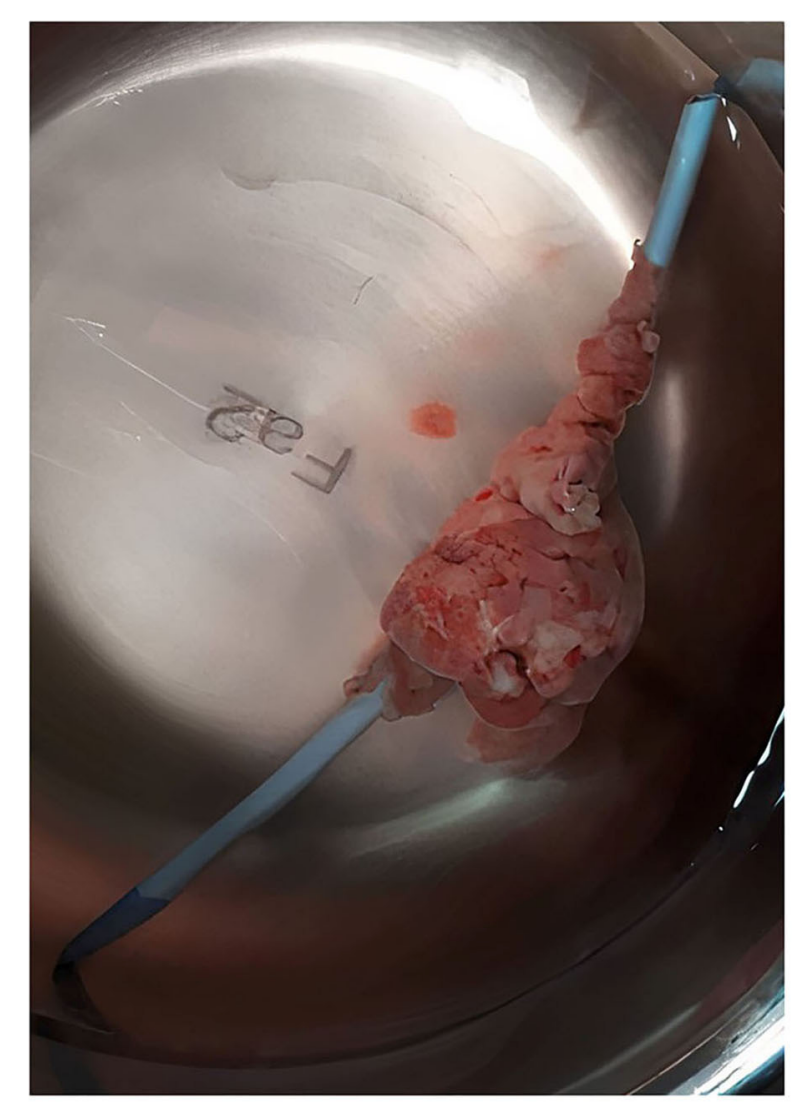

FIGURE 3 | Formation of a large thrombus around the CVC that was removed along with catheter via open heart surgery.

reported a lower mortality rate in the surgical intervention compared to the anticoagulation treatment. Meanwhile, anticoagulation treatment correlates with the theoretical risk of lysed clots lodging in pulmonary arteries and consequent pulmonary thromboembolism (13). In conclusion, there is an increasing concern about hypercoagulation and acute thrombosis in patients with COVID-19 infection. Therefore, conservative treatment with anticoagulation along with vigilant observation is recommended in all COVID19 patients to prevent subsequent hypercoagulation and thrombus formation.

\section{DATA AVAILABILITY STATEMENT}

The raw data supporting the conclusions of this article will be made available by the authors, without undue reservation.

\section{ETHICS STATEMENT}

This study involving human participant was reviewed and approved by Isfahan University of Medical Sciences (IR.MUI.MED.REC.1399.198). Written informed consent to 
participate in this study was provided by the participants' legal guardian/next of kin. Written informed consent was obtained from the individual(s), and minor(s)' legal guardian/next of kin, for the publication of any potentially identifiable images or data included in this article.

\section{REFERENCES}

1. Chen N, Zhou M, Dong X, Qu J, Gong F, Han Y, et al. Epidemiological and clinical characteristics of 99 cases of 2019 novel coronavirus pneumonia in Wuhan, China: a descriptive study. Lancet. (2020) 395:507-13. doi: 10.1016/S0140-6736(20)30211-7

2. Chen J, Fan H, Zhang L, Huang B, Zhu M, Zhou Y, et al. Retrospective analysis of clinical features in 101 death cases with COVID-19. medRxiv. (2020). doi: 10.1101/2020.03.09.20033068

3. Pan F, Ye T, Sun P, Gui S, Liang B, Li L, et al. Time course of lung changes on chest CT during recovery from 2019 novel coronavirus (COVID-19) pneumonia. Radiology. (2020) 295:715-21. doi: 10.1148/radiol.2020200370

4. $\mathrm{Hu} \mathrm{D}$, Liu K, Li B, Hu Z. Large intracardiac thrombus in a COVID19 patient treated with prolonged extracorporeal membrane oxygenation implantation. Eur Heart J. (2020) 41:3104-5. doi: 10.1093/eurheartj/eh aa524

5. Fried JA, Ramasubbu K, Bhatt R, Topkara VK, Clerkin KJ, Horn E, et al. The variety of cardiovascular presentations of COVID-19. Circulation. (2020) 141:1930-6. doi: 10.1161/CIRCULATIONAHA.120.0 47164

6. Karimi A, Rafiei, Tabatabaei S, Rajabnejad M, Pourmoghaddas Z, Rahimi $\mathrm{H}$, et al. An algorithmic approach to diagnosis and treatment of coronavirus disease 2019 (COVID-19) in children: Iranian Expert's Consensus Statement. Arch Pediatr Infect Dis. (2020) 8:e102400. doi: 10.5812/pedinfect.1 02400

7. Akhmerov A, Marbán E. COVID-19 and the heart. Circ Res. (2020) 126:144355. doi: 10.1161/CIRCRESAHA.120.317055

8. Connors JM, Levy JH. COVID-19 and its implications for thrombosis and anticoagulation. Blood. (2020). 135:2033-40. doi: 10.1182/blood.2020006000

\section{AUTHOR CONTRIBUTIONS}

All authors contributed to the analysis, interpretation of data, wrote the manuscript, approved the final version of the manuscript, and agreed to be accountable for all aspects of the work.

9. Marietta M, Ageno W, Artoni A, De Candia E, Gresele P, Marchetti M, et al. COVID-19 and haemostasis: a position paper from Italian Society on Thrombosis and Haemostasis (SISET). Blood Transfusion. (2020) 18:167. doi: 10.2450/2020.0083-20

10. Chen G, Wu D, Guo W, Cao Y, Huang D, Wang H, et al. Clinical and immunological features of severe and moderate coronavirus disease 2019. J Clin Invest. (2020) 130:2620-9. doi: 10.1172/JCI137244

11. McFadyen JD, Stevens H, Peter K. The emerging threat of (micro) thrombosis in COVID-19 and its therapeutic implications. Circ Res. (2020) 127:57187. doi: 10.1161/CIRCRESAHA.120.317447

12. Hussain N, Shattuck PE, Senussi MH, Velasquez Kho E, Mohammedabdul M, Sanghavi DK, et al. Large right atrial thrombus associated with central venous catheter requiring open heart surgery. Case Report Med. (2012) 2012:501303. doi: 10.1155/2012/501303

13. Negulescu O, Coco M, Croll J, Mokrzycki MH. Large atrial thrombus formation associated with tunneled cuffed hemodialysis catheters. Clin Nephrol. (2003) 59:40-6. doi: 10.5414/CNP59040

Conflict of Interest: The authors declare that the research was conducted in the absence of any commercial or financial relationships that could be construed as a potential conflict of interest.

Copyright (C) 2020 Bigdelian, Sedighi, Sabri, Dehghan, Pourmoghaddas, Saleh, Sherafat, Massoumi, Rezaei and Shahbazi. This is an open-access article distributed under the terms of the Creative Commons Attribution License (CC BY). The use, distribution or reproduction in other forums is permitted, provided the original author(s) and the copyright owner(s) are credited and that the original publication in this journal is cited, in accordance with accepted academic practice. No use, distribution or reproduction is permitted which does not comply with these terms. 